\title{
Transcription of Inflammatory Cytokine TNFa is Upregulated in Retinal Angiogenesis under Hyperoxia
}

\author{
Yuxi Feng ${ }^{a, b}$ Shalini Gross ${ }^{a, b} \quad$ Anupriya Chatterjee ${ }^{b} \quad$ Yumei Wang ${ }^{a} \quad$ Jihong Lin ${ }^{a}$ \\ Hans-Peter Hammes ${ }^{a}$ \\ ${ }^{a} 5^{\text {th }}$ Medical Clinic, Medical Faculty Mannheim, Heidelberg University, Mannheim, bInstitute of \\ Experimental and Clinical Pharmacology and Toxicology, Medical Faculty Mannheim, Heidelberg \\ University, Mannheim, Germany
}

\section{Key Words}

Angiogenesis $\cdot$ Hyperoxia $・$ Retina $・$ Hypoxia $\cdot$ TNF $\alpha$

\begin{abstract}
Background/Aims: Hypoxia induces angiogenesis while hyperoxia promotes vasoregression in the retina. We investigated herein the effect of prolonged hyperoxia on retinal angiogenesis and the underlying mechanism in an oxygen-induced retinopathy (OIR) model. Methods: Vascular morphology was quantified in whole-mount retina from the mice subjected to the conventional OIR model (c-OIR) or the OIR model with prolonged hyperoxia ( $p$-OIR). Expressions of genes related to angiogenesis were determined by real-time PCR. Results: p-OIR retinas showed few intraretinal neovascular tufts at the border of avascular zones, lacking preretinal neovascularization, whereas c-OIR retinas had numerous preretinal neovascularizations. $p$-OIR retinas demonstrated outgrowth of capillaries in the deep layers despite persistent hyperoxia and possess a larger avascular zone compared with the c-OIR retinas. The capillaries in the p-OIR retinas were well-formed in contrast to those in the c-OIR retinas. p-OIR retinas expressed significantly higher TNF $\alpha$ ( $\sim$ fold) than c-OIR retinas. The expression of vascular endothelial growth factor, Erythropoietin, Angiopoietin 1 and 2 remained unchanged. Conclusion: Our data demonstrate that TNF $\alpha$ transcription is increased in hyperoxia-promoted retinal angiogenesis, implicating it, in association with low VEGF levels, as a possible proponent in retinal angiogenesis under hyperoxia.
\end{abstract}

\section{Introduction}

Oxygen plays an essential role in physiological and pathological angiogenesis $[1,2]$. Hyperoxia and hypoxia can contribute to retinal vasoregression and neovascularization, respectively [3]. Consequences of oxygen tension in the retina can be studied in a conventional oxygen-induced retinopathy (c-OIR) mouse model [4]. In this model, new-born 
mice are placed into a hyperoxic environment $(75 \%)$ at p7, when the retinal superficial capillary layer has just reached completion. The consequence of hyperoxia is a suppression of physiological angiogenesis, resulting in formation of large central avascular zones in the retina. After the mice return to room air at $\mathrm{p} 12$, strong angiogenesis induced by multiple growth factors is triggered by hypoxia, promoting intraretinal vascular regeneration in the superficial and deep capillary layers, and preretinal neovascularization. VEGF, identified as a major contributor in regulating retinal angiogenesis in this model $[4,5]$, is produced by several retinal cells, most abundantly by glial cells (astrocytes and Müller cells) [6-8]. The importance of VEGF in regulation of retinal angiogenesis has been previously demonstrated by genetic gain- and loss-of-function experiments as well as pharmacological inhibition [911].

Apart from VEGF, other growth factors are involved in the vascular remodeling and angiogenesis in the retina. Epo is produced locally and regulated mainly by hypoxia, where it is found to be associated with both preretinal and intraretinal angiogenesis [1215]. It modulates the action of endothelial cells, inducing them to proliferate and mount an angiogenic response [16]. Additionally, the Ang-Tie2 system plays a pivotal role in hypoxiainduced retinal angiogenesis. Ang1 promotes transition of vessel to a mature network through pericyte recruitment via phosphorylation of receptor tyrosine kinase Tie2. In contrast, Ang2 is a natural antagonist of Ang1. Unlike Ang1, it is produced by endothelial and glial cells in the retina [17]. The vascular response to Ang2 is context dependent. In the presence of VEGF, it promotes sprouting angiogenesis and in its absence, causes vessel regression. Upregulation of Ang2 has been defined in the c-OIR model whereas Ang1 and Tie 2 are not regulated by hypoxia $[18,19]$. A 50\% reduction in the gene dosage of Ang2 leads to a decreased hypoxia-induced angiogenic response in the c-OIR model [20].

$\mathrm{TNF} \alpha$, a member of the cytokine family involved in immuno-inflammatory response, has both anti- and pro-angiogenic abilities [21]. Previous studies using deletion of TNF $\alpha$ revealed that it plays a role in preretinal neovascularization and revascularization of intraretinal vascular plexus in the c-OIR model [22].

Hyperoxia down-regulates VEGF in the c-OIR model and leads to vascular degeneration in the superficial capillary layer by apoptosis of endothelial cells, resulting in large avascular zones in the center of the retina. Vascular development is attenuated under hyperoxia in rodent retina models, but breakthrough angiogenesis appears possible by mechanisms which are yet unknown to date $[23,24]$. Therefore, we used a modified OIR model to assess retinal angiogenic response under hyperoxia, i.e. at low VEGF levels.

\section{Materials and Methods}

\section{Conventional OIR model}

Animal care and experiments were performed in accordance with the Association for Research in Vision and Ophthamology (ARVO) statement and were approved by the local government. Conventional OIR model (c-OIR) was performed in C57 BL6 mice as published previously [20]. It was established by postnatal subjection to $75 \%$ oxygen concentration for 5 days from $\mathrm{p} 7$ to $\mathrm{p} 12$ and was then returned from $\mathrm{p} 12$ to $\mathrm{p} 17$ to room conditions at relative hypoxia (Fig. 1). The eyes were then enucleated and stored immediately at $-80^{\circ} \mathrm{C}$.

\section{Modified OIR model}

In the modified OIR model (prolonged OIR model, p-OIR), mice were subjected to hyperoxia at $75 \%$ concentration oxygen from postnatal day 7 to day 17 (Fig. 1). The eyes were then enucleated and stored immediately at $-80^{\circ} \mathrm{C}$. Mice maintained at room air up to $\mathrm{p} 17$ served as controls.

Immunofluorescence staining

Retinal vasculature was visualized by staining with collagen type IV or Lectin. Pericytes were identified by staining with NG2. Eyes were fixed overnight in $4 \%$ formalin at $4^{\circ} \mathrm{C}$. On the second day, the retinas were dissected. The retinas were washed three times with PBS for 1 hour and incubated in blocking/ 


\section{Cellular Physiology Cell Physiol Biochem 2016;39:573-583 \\ \begin{tabular}{l|l} 
DOI: 10.1159/000445649 & $\begin{array}{l}\text { O 2016 The Author(s). Published by S. Karger AG, Basel } \\
\text { www.karger.com/cpb }\end{array}$
\end{tabular} \\ Feng et al.: Angiogenesis in the Hyperoxic Retina}

permeabilization buffer (1\% BSA, $0.5 \%$ Triton-100 in PBS) for 1 hour at room temperature. Retinas were incubated in Lectin or primary antibody diluted in blocking/permeabilization buffer overnight at $4^{\circ} \mathrm{C}$. After washing 3 times for 1 hour with PBS, the samples were alternatively incubated with secondary antibody or directly covered with mounting medium. For double staining, the retinas were further transferred to second pairs of primary and corresponding secondary antibody with the same procedure described above. Primary antibodies used were Lectin-TRITC (L5264, Sigma, Missouri, USA), or Lectin-FITC (L9381, Sigma, Missouri, USA), or rabbit anti Collagen IV (Bp8017, 1:200, Acris Antibodies GmbH, Herford, Germany), rabbit anti NG2 (AB5320, 1:200, CA, USA). Secondary antibodies used were swine anti-rabbit-TRITC (R0156, 1:20, DAKO Cytomation, Glostrup, Denmark), swine anti rabbit-FITC (F0205, 1:20, DAKO Cytomation, Glostrup, Denmark).

\section{Quantification of Avascular Zone}

The avascular zone is characterized by a region lacking in capillaries in the retinal center. Measurement of avascular and total retinal area was performed in retinas stained with vascular markers such as lectin or collagen IV. It was measured using a Leica DMRBE microscope connected to a video camera (Leica, Wetzlar, Germany).

\section{Quantification of Neovascular Tufts}

Neovascularization tufts were also quantitated in retinas stained with vascular marker as described above. They were characterized by their fist-like or glomerulus-like structure. The neovascularization tufts were quantitated with a depth of $230 \mu \mathrm{m}$ along the border of the avascular zone extending towards the vascularized area of the retina. Tufts beyond this depth towards the periphery of the retina were not included.

Quantification of sprout tips, arteriole tortuosity index and vascular tree

Sprout tips, arteriole tortuosity index, superficial and deep capillary diameter were quantitated in whole mount retinal staining with vascular marker as described above. The sprout tips are identified as finger-like projections, extending towards the avascular zone. Sprout tips were quantitated on the border of the avascular zone using a 20 times objective. Tortuosity index of the arterioles was quantified as described previously by Scott and colleagues [25]. Outgrowth of the deep vascular area was quantified in whole mount retina by measuring deep retinal vascular area. The value was standardized to the superficial retinal vascular area.

\section{RNA isolation and real time PCR}

Expression of genes associated with angiogenesis, such as VEGF, Angiopoeitin 1 and 2, EPO, TNF $\alpha$ was analyzed using the quantitative Taqman real time PCR. Retinal total RNA was extracted using TRIzol reagent (Invitrogen, Germany) according to the manufacturer's protocol. Reverse Transcription was performed with the QuantiTect RT kit from Qiagen (Qiagen GmbH, Germany). Samples were subjected to Taqman analysis using Taqman 2xPCR master Mix (Applied Biosystems, Weiterstadt, Germany). Expression of genes was analysed by $2^{-\triangle \Delta C T}$ method using ß-actin as housekeeping control [26]. Primers and probes were purchased from Applied Biosystems (Weiterstadt, Germany). VEGF: Mm01281449_m1; Ang1: Mm00456503_m1; Ang2: Mm00545822_m1; EPO: Mm01202754_g1; TNF: Mm00443258_m1.

\section{Statistical analysis}

Data are presented as mean \pm SEM. Unpairred t-test with Welch's correction or One-way ANOVA with Bonferonni's Multiple Comparison Post Test was performed using Graphpad Prism (San Diego California USA). A p-value less than 0.05 was considered statistically significant.

\section{Results}

Angiogenesis occurs despite hyperoxia

To investigate the existence and extent of angiogenesis under hyperoxia we used the p-OIR model (Fig. 1). During retinal development, at p7, blood vessels are present only in the superficial retinal layer. Hyperoxia in the c-OIR model between p7 to p12 induced 


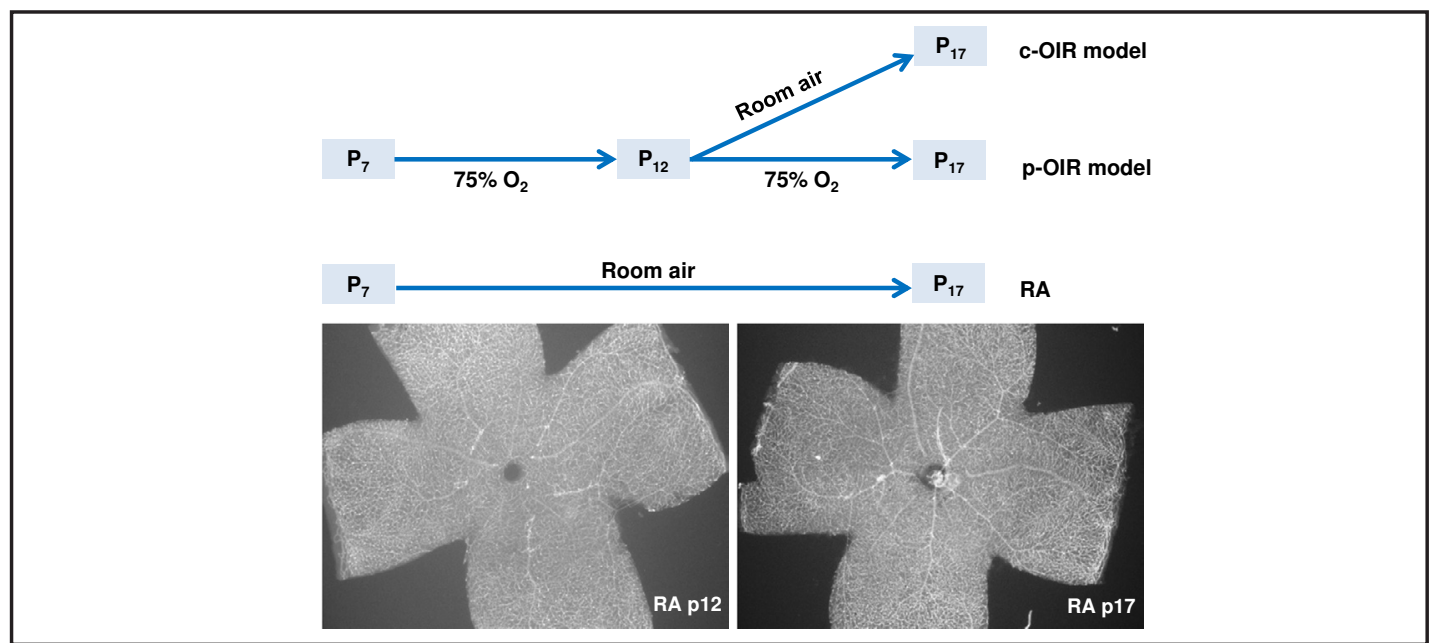

Fig. 1. Representation of experimental design. Mice at $\mathrm{p7}$ were subjected to hyperoxia at 75\% oxygen concentration till p17 constitute the p-OIR model. Mice at p7, initially subjected to hyperoxia at 75\% concentration, were moved to room air from p12 to p17, constituting the c-OIR model. These two models differ from p12 in the OIR model. Additional control animals at p17 were maintained at room air (RA). The normal developing vasculature in the retina at p12 and p17 are depicted in the lower panel.
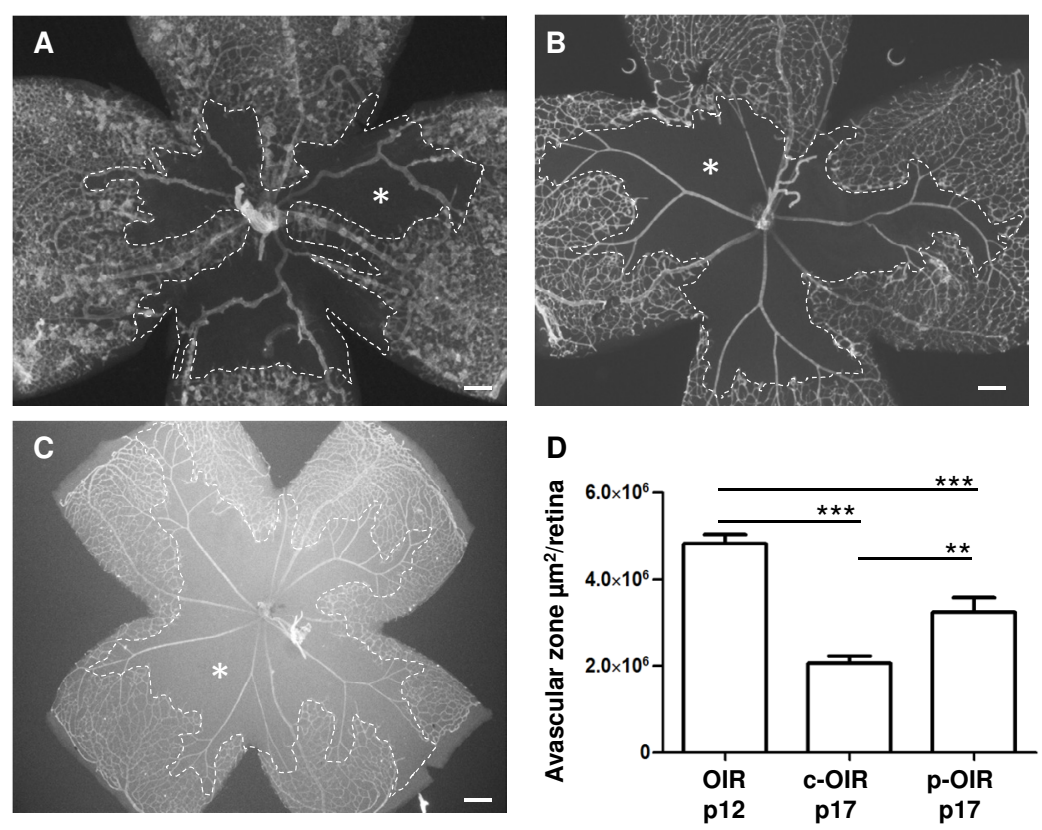

Fig. 2. Angiogenesis in the hyperoxic retina. $A$ and $B$ show a representative demonstration of retinal vascular network with a central avascular zone (AZ) at p17 in the c-OIR and p-OIR retina, respectively. A is focused on the preretinal neovascular tufts leaving intraretinal vessels out of focus. $\mathrm{C}$ demonstrates the $\mathrm{AZ}$ at $\mathrm{p} 12$ in the OIR model. No neovascularizations were detected. Both c-OIR and p-OIR retinas at p17 (A and B, respectively) show smaller AZ than that at p12 (C). A graphical comparison shows a larger AZ in the p-OIR retina than in the c-OIR retina at p17 (D). Retinas were stained with collagen IV. A: c-OIR retina at p17; B: p-OIR retina at p17; C: retinal vasculature at p12 in the OIR model. * in A-C: avascular zone labeled with dashed line. $\mathrm{n}=6-8,{ }^{* *} \mathrm{p}<0.01,{ }^{* * *} \mathrm{p}<0.001$. Scales in A-C: $200 \mu \mathrm{m}$.

regression of superficial retinal vessels, leading to formation of large avascular zone in the retinal center. There is no deep retinal capillary layer formed under hyperoxia in the c-OIR model at p12. After the mice returned to room air in the c-OIR model, retinal vessels regrew 


\section{Cellular Physiology Cell Physiol Biochem 2016;39:573-583 \begin{tabular}{ll|l} 
DOI: 10.1159/000445649 & $\begin{array}{l}\text { O 2016 The Author(s). Published by S. Karger AG, Basel } \\
\text { www.karger.com/cpb }\end{array}$
\end{tabular}

Fig. 3. Neovascular tufts are rare under hyperoxia. (A and B) are higher magnifications of the upper retinal leaves in Fig. $2 \mathrm{~A}$ and $\mathrm{B}$, respectively, showing neovascular tufts (arrows) at the border of the avascular zone. A is focused on the preretinal neovascular tufts leaving intraretinal vessels out of focus. (C and D) show neovascular tufts (arrows) and sprouts (arrowheads) in confocal microscopy images. E-G depict the quantification of the neovascularization (E), sprout tips (F) and arteriole tortuosity index (G) in the c-OIR and p-OIR retinas at $\mathrm{p} 17$. Rare neovascular tufts and lower arteriole tortuosity index were observed while more sprouts were found in the p-OIR retina compared with that in the c-OIR retina. A and $\mathrm{C}$ c-OIR retina at $\mathrm{p} 17$; $\mathrm{B}$ and $\mathrm{D}$ p-OIR retina at $\mathrm{p} 17$. $\mathrm{A}$ in $\mathrm{A}$ and $\mathrm{B}$ : arteriole; $\mathrm{V}$ in A and $B$ : venule. $n=6-8,{ }^{*} p<0.05 ;{ }^{*} p<$ $0.01{ }^{* * *} \mathrm{p}<0.001$. Scales in A and B: 400 $\mu \mathrm{m}$; Scales in C and D: $150 \mu \mathrm{m}$.

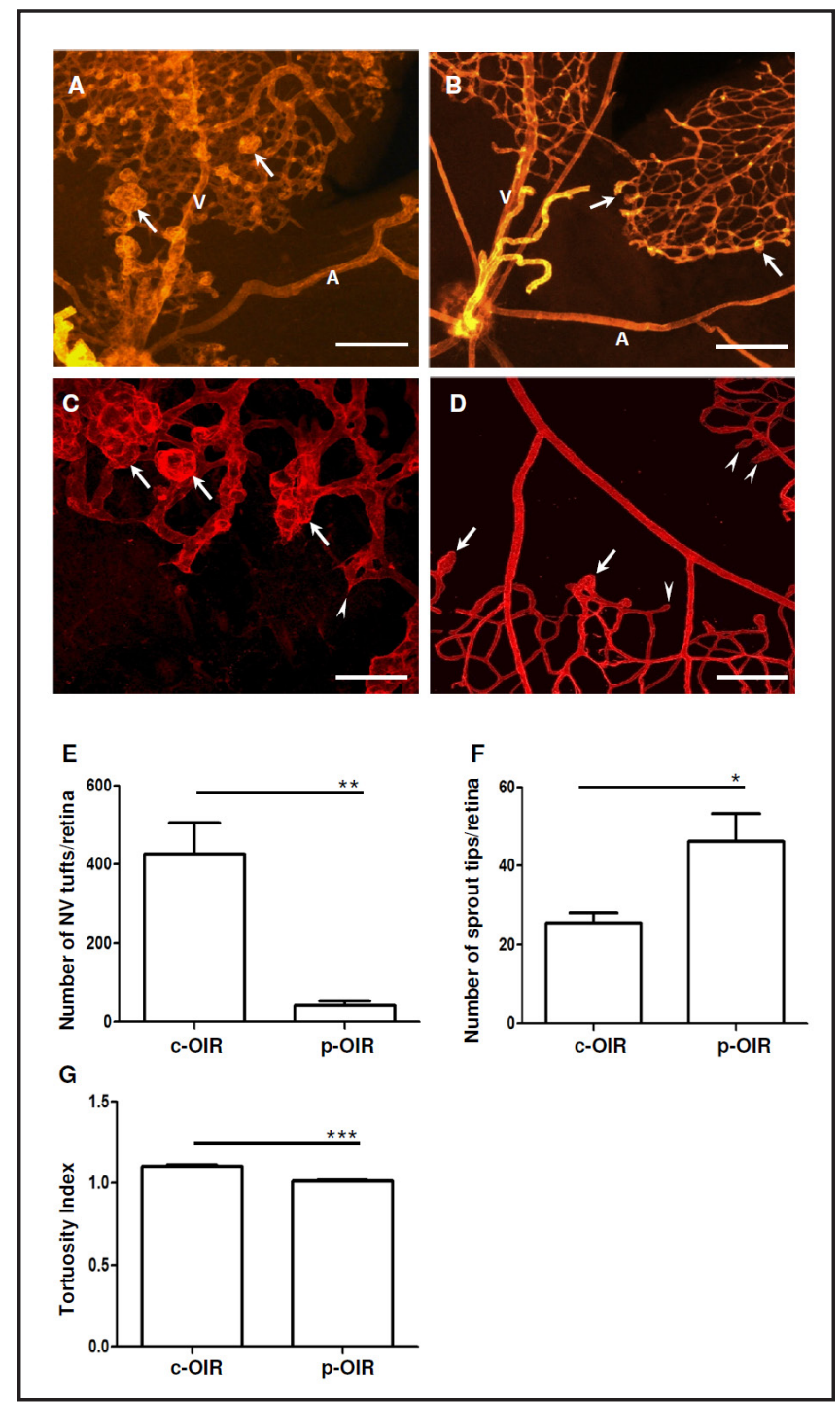

towards the avascular zone, and numerous preretinal neovascular tufts developed at p17. Theoretically, there must be no more vessels at p17 in the p-0IR model than at p12 in the retina under persistent hyperoxia. However, we observed new retinal vessels in the p-OIR retinas at p17. As shown in Fig. 2, retinal angiogenesis was observed in both p-OIR and c-OIR retinas under hyperoxia and hypoxia. c-OIR retinas at p17 showed a significantly smaller central avascular zone compared with p12 due to strong angiogenesis between p12 and p17 (Fig. 2A). Avascular zones persisted in the p-OIR retinas at p17 (Fig. 2B). However, compared with the OIR retinas at p12, p-OIR retinas demonstrated significantly smaller avascular zones at p17 ( $<<0.001$ ) (Fig. 2B). Nevertheless, p-OIR retinas displayed larger central avascular areas than c-OIR retinas at p17 (Fig. 2D, p < 0.01). The data suggest slower angiogenesis in hyperoxia between $\mathrm{p} 12$ and $\mathrm{p} 17$ in the p-OIR retinas towards the central avascular zones as compared with the c-OIR retinas.

\section{Well-organized vasculature during long-term hyperoxia}

We further analyzed in detail the vascular morphology in the c-OIR and p-OIR retinas at $\mathrm{p} 17$. The c-OIR retina demonstrated a chaotic structure of the vessel architecture, while the p-OIR retina exhibited a more regular vascular bed (Fig. 3). Numerous neovascular tufts (arrows) were clustered or fused to capillaries and venules in particular (Fig. 3A and C). We observed a few neovascular tufts in the p-OIR retina localized at the border of the AZ as well 


\section{Cellular Physiology Cell Physiol Biochem 2016;39:573-583 and Biochemistry Published online: July 11, $2016 \quad \begin{aligned} & \text { DOI: 10.1159/000445649 } 2016 \text { The Author(s). Published by S. Karger AG, Basel } \\ & \text { www.karger.com/cpb }\end{aligned}$

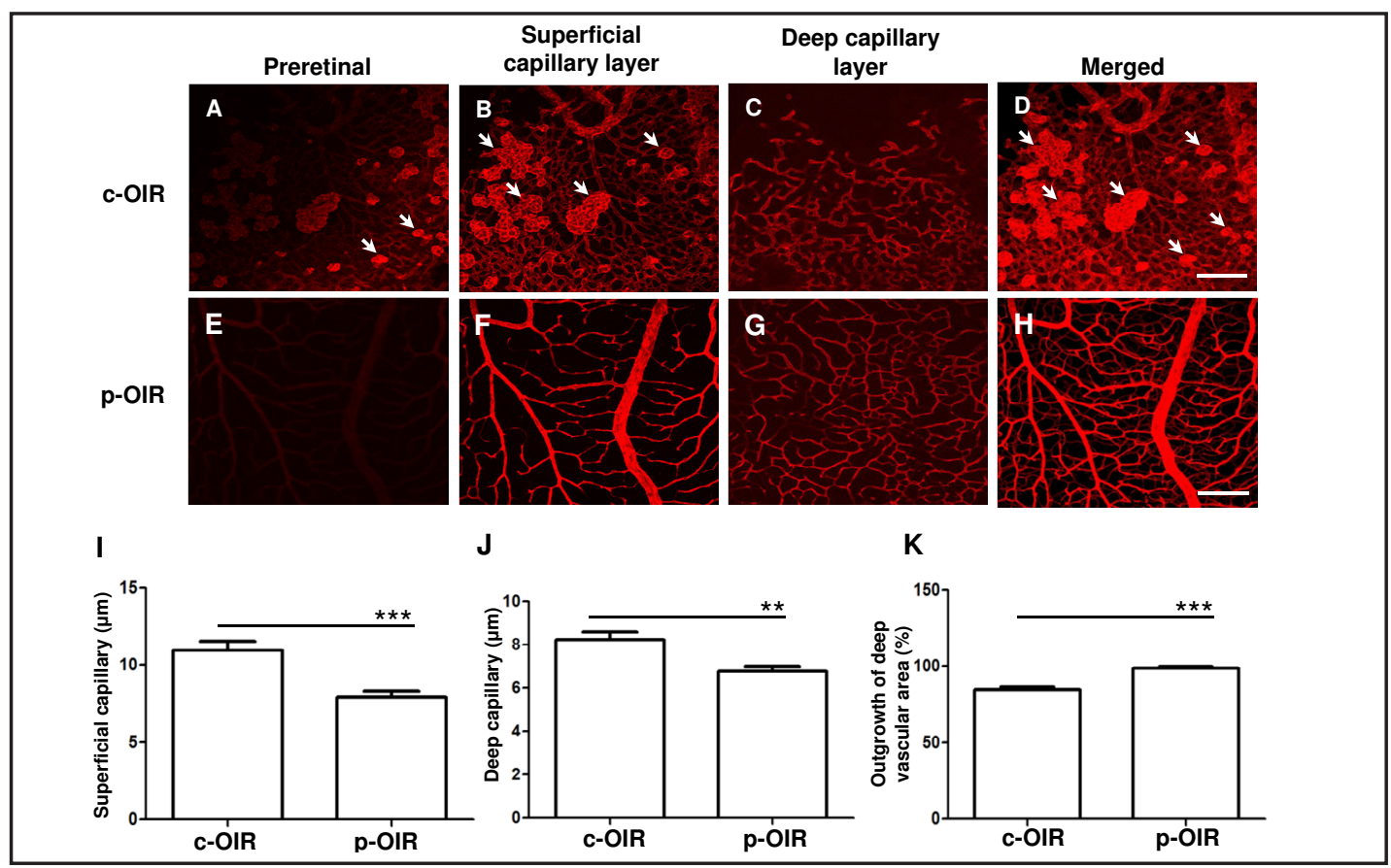

Fig. 4. Retinal vessels formed under hyperoxia are well-organized. (A and E) Preretinal region. Preretinal location in the c-OIR retinas shows numerous neovascular tufts (A) while no neovascularizations were detected in the p-OIR retinas (E). (B and F) Retinal superficial capillary layer. Neovascularizations were frequently seen in the c-OIR retina (B) compared to well-ordered capillary network development in the p-OIR retina (F). (C and G) Retinal deep capillary layer. The vessels in the c-OIR retinas were partially formed and disorganized, giving the appearance of an incomplete network (C). The vessels in the p-OIR retina, on the other hand, showed well-organized, narrow capillary formation (G). D and H: Merged images from preretinal to deep capillary layer of the c-OIR and p-OIR retinas. Scales in D and H: $150 \mu \mathrm{m}$. Figure I, J and K are graphical representations of superficial capillary diameter, deep capillary diameter and deep vascular outgrowth (displayed in ratio to superficial vascular outgrowth), respectively, in the c-OIR and p-OIR retinas. Arrows indicate neovascular tufts. $\mathrm{n}=6-8,{ }^{* *} \mathrm{p}<0.01{ }^{* * *} \mathrm{p}<0.001$.

(Fig. 3B and D). Quantification of neovascular tufts verified our observation showing that p-OIR retinas developed significantly lesser neovascular tufts than in the c-OIR retinas (Fig. $3 \mathrm{E}, \mathrm{p}<0.01$ ), suggesting moderate angiogenesis. Quantification of sprout tips (arrowheads) at the border of avascular zones (Fig. 3C and D), displayed a high number of sprout tips in the p-OIR retinas in comparison with the c-OIR retinas (Fig. 3F. p < 0.05). Arteriolar tortuosity has been identified as an indicator of severity of hypoxia and angiogenic response in the c-OIR model. It predicts the appearance of retinal neovascularization in this model. Therefore, we further analyzed the arteriolar tortuosity in these two models. No arteriolar tortuosity was observed in the p-OIR retinas (Fig. 3G).

We further examined the capillaries in the p-OIR retinas. Confocal microscope images confirmed presence of preretinal neovascular tufts in the c-OIR model (Fig. 4A) as well as intraretinal tufts in the superficial retinal capillary layer (Fig. 4B). No preretinal neovascular tufts were found in the p-OIR retina (Fig. 4E). Neovascular tufts in the c-OIR model originated mainly from the superficial capillaries that were dense, irregular and disorganized. In contrast to c-OIR retinas, established retinal superficial vessels in the p-OIR model appear much more regular and well-arranged. The diameters of the superficial capillary layers of the p-OIR retinas were significantly lesser than in the c-OIR retinas (Fig. 4I, p < 0.001). Neither vessel tortuosity nor neovascular tuft was detected. Unexpectedly, we found formation of deep capillaries in the p-OIR retinas at p17. As shown in Fig. 4G and H, the deep capillary layers in the p-OIR retinas had developed to the same extent as the superficial capillary layer, whereas the deep capillary layers had only partially developed beneath the superficial 


\section{Cellular Physiology and Biochemistry

Fig. 5. Vessels formed in hyperoxia are covered by pericytes. Representative demonstration of the pericyte coverage in the central and peripheral retinal vasculature of the c-OIR and pOIR mice. p-OIR retinas show normal distribution of pericytes on the retinal vessels. Scale bars: $75 \mu \mathrm{m}$.

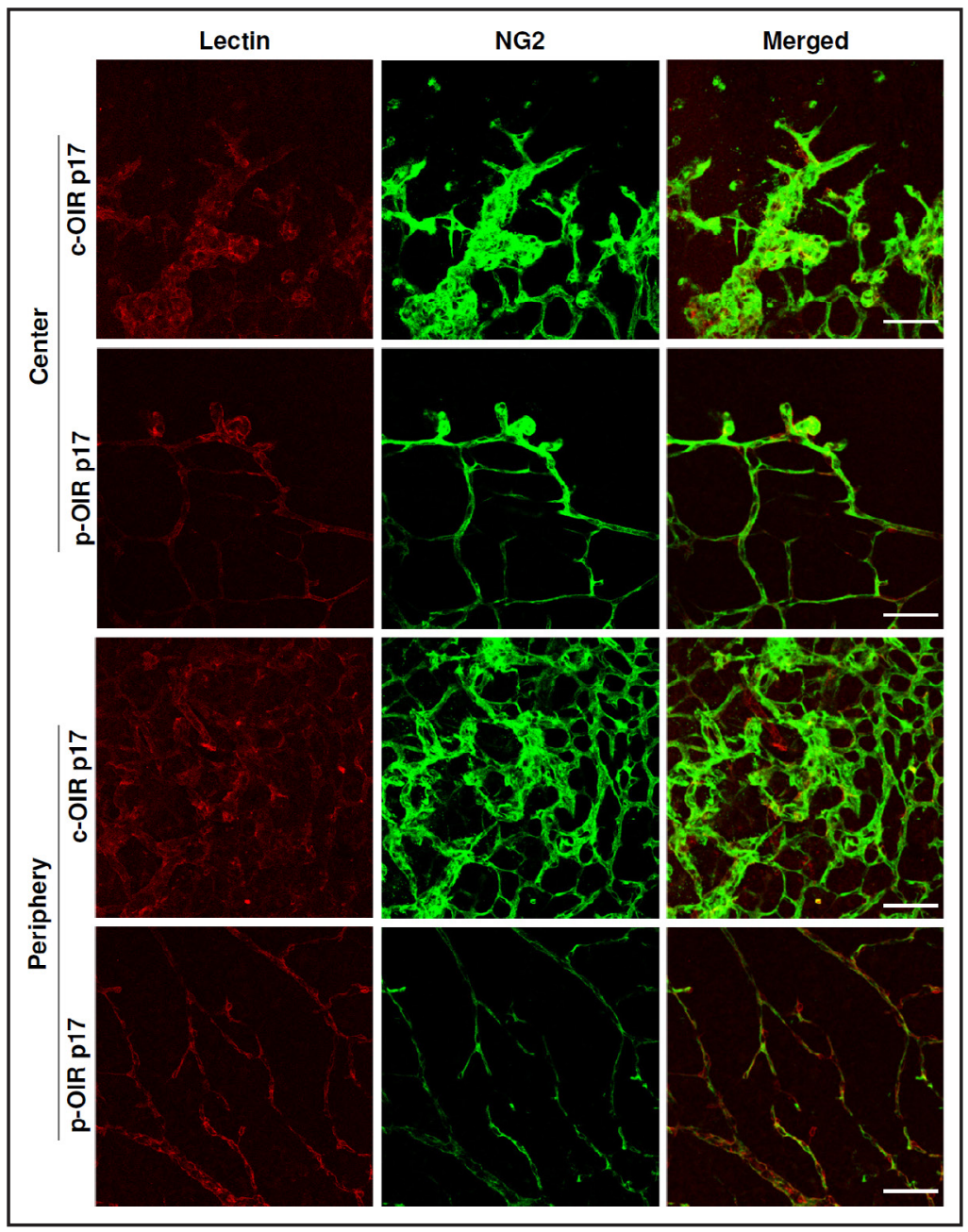

capillary layers in the c-OIR retinas (Fig. $4 \mathrm{C}$ and $4 \mathrm{~K}, \mathrm{p}<0.001$ ). The deep capillary vessels in the p-OIR retinas appeared to form a regular and well-organized vascular network, while in the c-OIR retinas capillaries were dilated and irregular (Fig. 4C and 4G). Quantitation revealed significantly higher capillary diameters in the p-OIR retinas (Fig. 4J, p < 0.01). The data suggest that angiogenesis occurs under hyperoxia despite the lack of angiogenic stimuli and the resulting newly-formed vessels are highly organized. No vessels were observed in the outer plexiform layer and outer nuclear layer of the retina (data not shown).

Pericyte coverage plays an essential role in vascular development. Thus, we assessed the pericyte coverage by immunofluorescence staining for the pericyte marker NG2 in the p-OIR retinas at $\mathrm{p} 17$. As depicted in Fig. 5, pericytes were clustered on the vessels including sprouts at the border of the avascular zone in both OIR retinas. In the c-OIR retinas, pericytes additionally convened at the neovascular tufts and in the capillaries close to the neovascular tufts. In contrast, pericytes were regularly distributed in the p-OIR retinal capillaries. Difference in distribution of pericyte coverage in the c- and p-OIR retinas might be the result of variation in vascular remodeling.

$T N F \alpha$ is increased in the development of retinal deep capillary layer under hyperoxia

We assessed gene expression of relevant angiogenic factors by real time PCR (Fig. 6). In the c-OIR model, VEGF level at p17 was elevated compared to the controls (RA) which were kept in room air, not subjected to either OIR model. In the p-OIR model VEGF expression was suppressed significantly in comparison with that in the c-OIR model, similar to the RA control retinas. The data provide clear evidence that angiogenesis occurred in the hyperoxic 
Fig. 6. $\mathrm{TNF} \alpha$ is upregulated during retinal angiogenesis under hyperoxia. The illustrations show gene expression of VEGF, Ang1, Ang2, Epo and TNF $\alpha$ in the c-OIR, p-OIR and room air (RA) retinas at p17. $\operatorname{VEGF}(\mathrm{A})$, Ang-2 (C) and Epo (D) levels were upregulated in the c-OIR retinas but remained unchanged in the $p$ OIR retinas compared with RA retinas. Ang1 levels remained unaffected by changes in oxygen concentration (B). TNF $\alpha$ was upregulated in the p-OIR retinas compared to the c-OIR and $\mathrm{RA}$ retinas (E). RA: retinas from mice kept in room air. $\mathrm{n}=6,{ }^{* *} \mathrm{p}<0.01, * * * \mathrm{p}<0.001$.

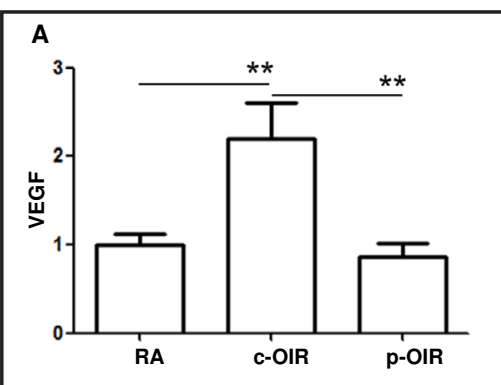

C
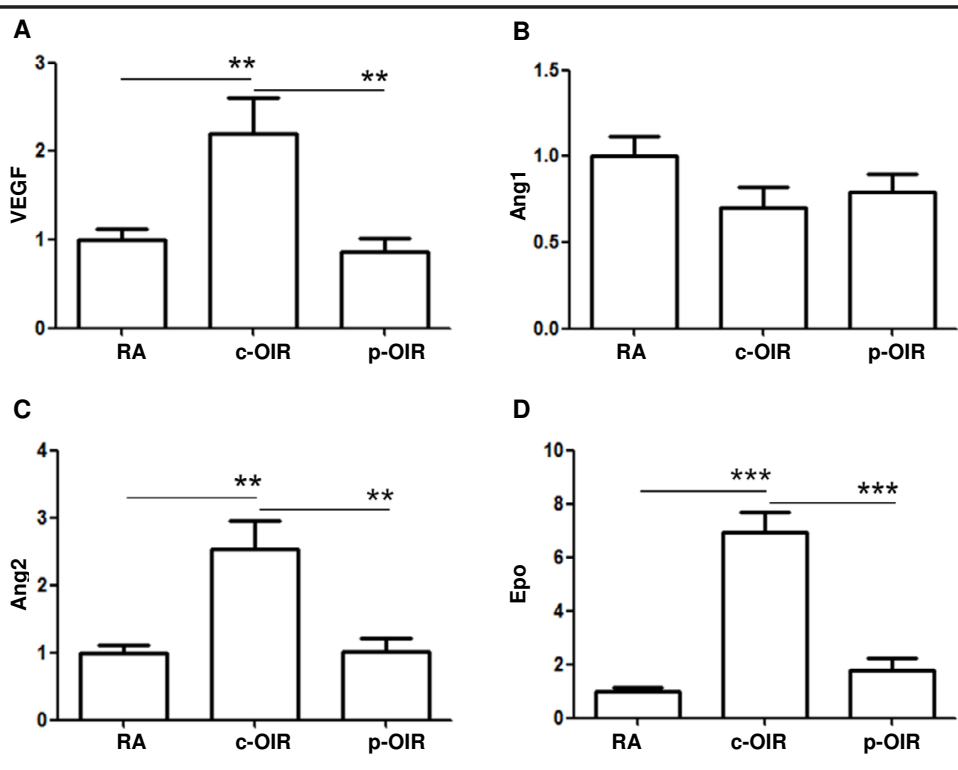

D

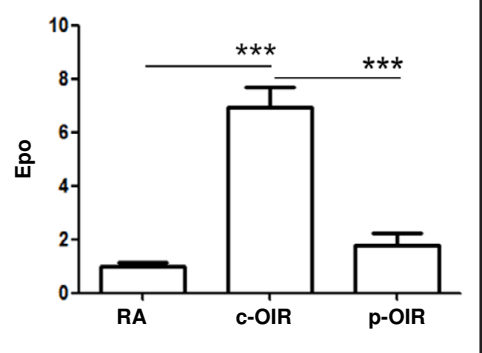

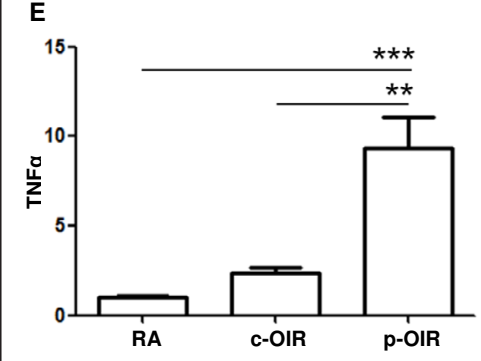

retinas despite low VEGF levels. Thus, angiogenesis was not driven by VEGF in the p-OIR model, suggesting involvement of other factors which led to the angiogenic response in persistent hyperoxia. Ang1 gene expression showed no significant alteration in retinas of the c-OIR model, p-OIR model and RA. This suggest that Ang1 is unaffected by oxygen concentrations. In the c-OIR model, Ang2 levels were elevated compared to RA controls. In the p-OIR model, its expression was low under long-term hyperoxia and did not differ from the RA mice. Thus, Ang2 is also not pivotal in the angiogenic response in the p-OIR retina under persistent hyperoxia. Epo was highly up regulated at p17 in the c-OIR model. In contrast, Epo was not upregulated to a significant level in the p-OIR model. TNF $\alpha$, a key regulator of the inflammatory response in angiogenesis, was elevated at p17 in the c-OIR model. In the p-OIR model, TNF $\alpha$ was 9 - and 4 folds upregulated compared to controls and c-OIR retinas, respectively. The data indicate that TNF $\alpha$ may play as a possible proponent in association with low VEGF levels in retinal angiogenesis under prolonged hyperoxia.

\section{Discussion}

Our study demonstrated that retinal angiogenesis persists despite low VEGF levels under hyperoxia. The forming vessels appear regular and well-organized. During hyperoxia, under low VEGF, Epo and Ang2, increased TNF $\alpha$ may play a possible proponent in retinal angiogenesis, especially the formation of well-organized retinal deep capillary network.

\section{Angiogenesis despite hyperoxia}

Our study identified retinal angiogenesis under persistent hyperoxia and revealed that angiogenesis proceeds not only under hypoxia but also hyperoxia. It appears that the eye in hyperoxia represents an exclusive condition as there is no other organ known in which 
hyperoxia induces angiogenesis. The strong hyperoxic condition induced in our model is unique and different from any physiological hyperoxic condition, as the levels of oxygen used here are substantially higher. Therefore, hyperoxia-induced angiogenesis must differ from physiological angiogenesis, in particular by the relative balance of active growth factors. Physiological angiogenesis is linked to VEGF, Epo as well as angiopoietins, but rarely associated to TNF $\alpha$. Hypoxia is able to induce expression of VEGF, Epo, Ang2 and TNF $\alpha$. In contrast, hyperoxia-induced retinal angiogenesis in this study represents the balance of high TNF- $\alpha$ and low hypoxia-induced growth factors such as VEGF, Epo and Ang2. Hyperoxia has been conventionally believed to suppress VEGF expression, leading to vascular degeneration $[5,27]$. In our study, we provide evidence showing although VEGF levels in the retina was not altered, retinal vessels developed despite persistent hyperoxia. Our observation is consistent with previous studies showing breakthrough revascularization under hyperoxia $[23,24]$. However, the underlying mechanisms remain to be illuminated. Promoted retinal angiogenesis under hyperoxia in our study was observed in both superficial and deep capillary layer. Of note, hyperoxia induced a more uniform growth of vasculature in the deep capillary layer than under hypoxia. Our data suggest the existence of factors besides VEGF, which are involved in angiogenesis under hyperoxia.

\section{Angiogenesis under hyperoxia differs from that under hypoxia}

Moreover, we found that the angiogenesis under hyperoxia differs from that under hypoxia. Firstly, hypoxia in the c-OIR model between p12 to p17 is a strong promoter for angiogenesis. Vessels grow quickly and irregularly, and strong preretinal angiogenesis occurs. In contrast, under persistent hyperoxia as in the p-OIR model from p12 to p17, vessels are generated gradually, indicated by a large avascular zone, but well-organized, with intraretinal instead of preretinal vessels. Larger avascular zone in the p-OIR model compared with that in the c-OIR model suggests that persistent hyperoxia provokes a moderate rather than dramatic effect on angiogenesis. Secondly, in comparison with c-OIR retinas, the p-OIR retinas had more sprouting tips, but fewer neovascularization tufts intraretinally. It indicates that an angiogenic demand is required under persistent hyperoxia, but, a weak angiogenic effect is exerted due to lack of appropriate angiogenic ambience such as insufficient growth factor VEGF. Thus, the angiogenic response in long-term hyperoxia is restricted intraretinally. Thirdly, the angiogenesis-driving factor in hypoxia and hyperoxia differ in VEGF for the c-OIR model and TNF $\alpha$ with low VEGF for the p-OIR model, respectively. The strong elevation of VEGF promotes predominant angiogenic response in the retinal superficial capillary layer in the c-OIR model, while elevated TNF $\alpha$ in association with low VEGF levels may induce an angiogenic response in the deep capillary layer of the p-OIR model.

\section{Implication of TNF $\alpha$ upregulation in retinal angiogenesis under hyperoxia}

Previous publications revealed that the retinal deep capillary layer is formed from angiogenesis that is guided by molecules produced in Müller cells [28]. TNF $\alpha$ was found to be produced by several cell types in the retina, such as neuronal cell, Müller cells, microglia cells and endothelial cells $[29,30]$. Reports regarding the role of TNF $\alpha$ in angiogenesis are contradictory. In vitro experiments demonstrated that TNF $\alpha$ acts as an anti-angiogenic cytokine inhibiting endothelial proliferation and capillary-like tube formation [31]. There is growing body of evidence showing that, in vivo, TNF $\alpha$ is a pro-angiogenic factor. In the c-OIR model, pro-angiogenic role of TNF $\alpha$ has been described by Yossuck et al. [32]. Gardiner and colleagues disclosed that, despite the existence of reduced preretinal angiogenesis, accelerated intraretinal angiogenesis in the c-OIR model during hypoxic phase was described upon TNF $\alpha$ inhibition [22]. The present study revealed that development of deep retinal vascular network is concomitant with upregulation of pro-angiogenic TNF $\alpha$ in the retina, in accordance with observations showing the decelerated angiogenesis by TNF $\alpha$ inhibitors. The possible correlation between TNF $\alpha$ elevation and well-organized retinal deep capillary layer may present a novel concept concerning importance of the inflammatory cytokine cascade for retinal deep capillary formation in contrast to classical growth factors like VEGF. 


\section{Cellular Physiology Cell Physiol Biochem 2016;39:573-583 \begin{tabular}{l|l} 
and Biochemist 10.1159/000445649 & $\begin{array}{l}\text { D) 2016 The Author(s). Published by S. Karger AG, Basel } \\
\text { www.karger.com/cpb }\end{array}$ \\
\hline
\end{tabular} \\ Feng et al.: Angiogenesis in the Hyperoxic Retina}

The most predominant growth factor identified for retinal vessel development is VEGF, both in physiological and pathological retinal angiogenesis. Depletion of growth factors interrupts retinal physiological and pathological angiogenesis [27, 33]. In our study, no changes were detected in VEGF, Epo and Ang2 expression in the p-OIR model. Constitutive retinal VEGF, Ang1, Ang2 and Epo levels seem to be required for basic maintenance and remodeling of the retinal vasculature. In contrary to promotion of traditional growth factors, we identified an increase in TNF $\alpha$ level in the p-OIR retinas. Herein, TNF $\alpha$ may contribute as an inflammatory mediator and a pro-angiogenic factor in retinal angiogenesis. Hyperoxic environment may attract likely cells in the retina to release TNF $\alpha$. However, it is not excluded that other inflammatory cytokines are also activated under hyperoxia. This remains to be assessed. Further studies of retinal angiogenesis in the OIR model by blocking TNF signaling in inducible $\mathrm{TNF} \alpha$ knockout mouse might explore the important role of $\mathrm{TNF} \alpha$ under hyperoxia.

In summary, angiogenesis develops in the retina under hyperoxia. The spread of wellorganized retinal deep capillary layer during hyperoxic phase underlines the possible proangiogenic effect of TNF $\alpha$. The underlying mechanisms require further investigation.

\section{Acknowledgements}

The study was supported by the DFG, DDG and GRK. The authors would like to thank N. Dietrich, W. Schwarz, U. Kaiser and P. Bugert greatly for their technical assistance.

\section{Disclosure Statement}

None.

\section{References}

1 Fraisl P, Mazzone M, Schmidt T, Carmeliet P: Regulation of angiogenesis by oxygen and metabolism. Dev Cell 2009;16:167-179.

2 Pugh CW, Ratcliffe PJ: Regulation of angiogenesis by hypoxia: role of the HIF system. Nat Med 2003;9:677684.

3 Stahl A, Connor KM, Sapieha P, Chen J, Dennison RJ, Krah NM, Seaward MR, Willett KL, Aderman CM, Guerin KI, Hua J, Lofqvist C, Hellstrom A, Smith LE: The mouse retina as an angiogenesis model. Invest Ophthalmol Vis Sci 2010;51:2813-2826.

4 Scott A, Fruttiger M: Oxygen-induced retinopathy: a model for vascular pathology in the retina. Eye (Lond) 2010;24:416-421.

5 Smith LE, Wesolowski E, McLellan A, Kostyk SK, D'Amato R, Sullivan R, D'Amore PA: Oxygen-induced retinopathy in the mouse. Invest Ophthalmol Vis Sci 1994;35:101-111.

6 Fruttiger M: Development of the retinal vasculature. Angiogenesis 2007;10:77-88.

7 Eichler W, Kuhrt H, Hoffmann S, Wiedemann P, Reichenbach A: VEGF release by retinal glia depends on both oxygen and glucose supply. Neuroreport 2000;11:3533-3537.

8 Gerhardt H, Golding M, Fruttiger M, Ruhrberg C, Lundkvist A, Abramsson A, Jeltsch M, Mitchell C, Alitalo K, Shima D, Betsholtz C: VEGF guides angiogenic sprouting utilizing endothelial tip cell filopodia. J Cell Biol 2003;161:1163-1177.

9 Heidary G, Vanderveen D, Smith LE: Retinopathy of prematurity: current concepts in molecular pathogenesis. Semin Ophthalmol 2009;24:77-81.

10 Neufeld G, Cohen T, Gengrinovitch S, Poltorak Z: Vascular endothelial growth factor (VEGF) and its receptors. FASEB J 1999;13:9-22.

11 Sone H, Kawakami Y, Segawa T, Okuda Y, Sekine Y, Honmura S, Suzuki H, Yamashita K, Yamada N: Effects of intraocular or systemic administration of neutralizing antibody against vascular endothelial growth factor on the murine experimental model of retinopathy. Life Sci 1999;65:2573-2580. 


\section{Cellular Physiology Cell Physiol Biochem 2016;39:573-583 \begin{tabular}{l|l|l}
\hline DOI: 10.1159/000445649 & $\begin{array}{l}\text { ( ) 2016 The Author(s). Published by S. Karger AG, Basel } \\
\text { www.karger.com/cpb }\end{array}$
\end{tabular} \\ Feng et al.: Angiogenesis in the Hyperoxic Retina}

12 Alnaeeli M, Wang L, Piknova B, Rogers H, Li X, Noguchi CT: Erythropoietin in brain development and beyond. Anat Res Int 2012;2012:953264.

13 Chen J, Connor KM, Aderman CM, Willett KL, Aspegren OP, Smith LE: Suppression of retinal neovascularization by erythropoietin siRNA in a mouse model of proliferative retinopathy. Invest Ophthalmol Vis Sci 2009;50:1329-1335.

14 Garcia-Ramirez M, Hernandez C, Simo R: Expression of erythropoietin and its receptor in the human retina: a comparative study of diabetic and nondiabetic subjects. Diabetes Care 2008;31:1189-1194.

15 Mowat FM, Gonzalez F, Luhmann UF, Lange CA, Duran Y, Smith AJ, Maxwell PH, Ali RR, Bainbridge JW: Endogenous erythropoietin protects neuroretinal function in ischemic retinopathy. Am J Pathol 2012;180:1726-1739.

16 Ribatti D, Presta M, Vacca A, Ria R, Giuliani R, Dell'Era P, Nico B, Roncali L, Dammacco F: Human erythropoietin induces a pro-angiogenic phenotype in cultured endothelial cells and stimulates neovascularization in vivo. Blood 1999;93:2627-2636.

17 Hanahan D: Signaling vascular morphogenesis and maintenance. Science 1997;277:48-50.

18 Feng Y, Vom Hagen F, Wang Y, Beck S, Schreiter K, Pfister F, Hoffmann S, Wagner P, Seeliger M, Molema G, Deutsch U, Hammes HP: The absence of angiopoietin-2 leads to abnormal vascular maturation and persistent proliferative retinopathy. Thromb Haemost 2009;102:120-130.

19 Sato T, Kusaka S, Hashida N, Saishin Y, Fujikado T, Tano Y: Comprehensive gene-expression profile in murine oxygen-induced retinopathy. Br J Ophthalmol 2009;93:96-103.

20 Feng Y, Wang Y, Pfister F, Hillebrands JL, Deutsch U, Hammes HP: Decreased hypoxia-induced neovascularization in angiopoietin-2 heterozygous knockout mouse through reduced MMP activity. Cell Physiol Biochem 2009;23:277-284.

21 Frater-Schroder M, Risau W, Hallmann R, Gautschi P, Bohlen P: Tumor necrosis factor type alpha, a potent inhibitor of endothelial cell growth in vitro, is angiogenic in vivo. Proc Natl Acad Sci USA 1987;84:52775281.

22 Gardiner TA, Gibson DS, de Gooyer TE, de la Cruz VF, McDonald DM, Stitt AW: Inhibition of tumor necrosis factor-alpha improves physiological angiogenesis and reduces pathological neovascularization in ischemic retinopathy. Am J Pathol 2005;166:637-644.

23 Dorfman A, Dembinska O, Chemtob S, Lachapelle P: Early manifestations of postnatal hyperoxia on the retinal structure and function of the neonatal rat. Invest Ophthalmol Vis Sci 2008;49:458-466.

24 Gu X, Samuel S, El-Shabrawey M, Caldwell RB, Bartoli M, Marcus DM, Brooks SE: Effects of sustained hyperoxia on revascularization in experimental retinopathy of prematurity. Invest Ophthalmol Vis Sci 2002;43:496-502.

25 Scott A, Powner MB, Fruttiger M: Quantification of vascular tortuosity as an early outcome measure in oxygen induced retinopathy (OIR). Exp Eye Res 2014;120:55-60.

26 Livak KJ, Schmittgen TD: Analysis of relative gene expression data using real-time quantitative PCR and the 2(-Delta Delta C(T)) Method. Methods 2001;25:402-408.

27 Alon T, Hemo I, Itin A, Pe'er J, Stone J, Keshet E: Vascular endothelial growth factor acts as a survival factor for newly formed retinal vessels and has implications for retinopathy of prematurity. Nat Med 1995;1:1024-1028.

28 Dorrell MI, Friedlander M: Mechanisms of endothelial cell guidance and vascular patterning in the developing mouse retina. Prog Retin Eye Res 2006;25:277-295.

29 Tezel G, Li LY, Patil RV, Wax MB: TNF-alpha and TNF-alpha receptor-1 in the retina of normal and glaucomatous eyes. Invest Ophthalmol Vis Sci 2001;42:1787-1794.

30 Wang AL, Yu AC, He QH, Zhu X, Tso MO: AGEs mediated expression and secretion of TNF alpha in rat retinal microglia. Exp Eye Res 2007;84:905-913.

31 Marchetti M, Vignoli A, Russo L, Balducci D, Pagnoncelli M, Barbui T, Falanga A: Endothelial capillary tube formation and cell proliferation induced by tumor cells are affected by low molecular weight heparins and unfractionated heparin. Thromb Res 2008;121:637-645.

32 Yossuck P, Yan Y, Tadesse M, Higgins RD: Dexamethasone alters TNF-alpha expression in retinopathy. Mol Genet Metab 2001;72:164-167.

33 Carmeliet P: Angiogenesis in life, disease and medicine. Nature 2005;438:932-936. 